\title{
Zur Syphilis-Statistik
}

Bisiang sind die Versuche, eine allgemeine Statistik der syphilitischen Krankheiten zu schaffen, nicht über den Anfang hinausgelangt. Somit kann es kein Wunder nehmen, wenn die massgebenden Faktoren je nach dem ver-schiedenen Standpunkte eine Über- oder eine Unterschätzung der tatsäch-lichen Verhältnisse bevorzugen. Um so aussichtsvoller scheint der Hinblick auf bestimmte Schiehten der Bevölkerung, seien dieselben nach dem Stande oder nach der örtlichen Übersicht begrenzt. Mitteilungen dieser Art müssen als willkominene Bausteine zur Fundamentierung zukünftigen Wissens be-trachtet werden. So auch ein Vortrag, den in der Wiener Dermatologischen Gesellschaft unlängst Stabsarzt Dr. Buraczynski über „die venerischen 276 Zur Syphilis-Statistik.

Erkrankungen und deren Prophylaxe in der Armee" gehalten hat. Danach entfallen auf die Osterreichisch-Ungarische Armee jährlích durchschnittlicb 18000 Fälle venerischer Erkrankungen, das ist 6 pCt. der gesamten Er-krankungen pro Jahr, davon 2/6 Lues und 1/6 Ulcus molle. Der grösste Zugang tritt ein im Oktober mit Einrücken der Rekruten, und höchst versehieden ist die Erkrankungs-Ziifer in den einzelnen Garnisonen, je naeh der strengeren oder nachlässigerea Kontrolle der Prostituierten. Jedoch er-scheinen die Zahlen noch zu gering, da manehe Patienten wegen sonstiger Krankheiten auf anderen Abteilungen behandelt werden oder sich der dienst-lichen Erkennung zu entziehen wissen. Enipfohlen werden besonders Instruktionsstunden fiir einrückende Rekruten und Reservisten, sowie wissen-schaftliche Vorträge in den Kadettenschulen der Militär-Akademie und die Untersuchung bei KontrollVersammlungen. Da doch jeder einzelne Pall nicht nur einen Erfolg fiir sich bedeutet, sondern seine $\mathrm{P}>$ ehandlung auch die Verstopfung weiterer Injektionsquellen mit sich bringt, sehen wir in diesen Auslassungen ein Interesse, welches von militär-ärztlicher Seite der Einschränkuøg der Geschlechtskrankheiten entgegengebracht wird.

So gibt uns der „Bericht über die Geschlechtskrankheiten in Kiel und Umgegend” in dem Zeitraum vom 1. September 1901 bis obendahin 1903, erstattet von Dr. C. Schirren in Kiel, eine bemerkenswerte Auf-klärung über das Auftreten der Venerie in einem übersichtlichen Eezirke. Die Zusammenstellung ist auf Grund von Zãhlblättern erfolgt, die von einei* Anzahl, wenn auch nicht von alien Arzten ausgefüllt sind. Die in Frage kommende Bevölkerung beträgt 164408 Einwohner. Auf diese Zahl kommen 3276 Geschlechtskranke, von denen 920 syphilitisch und 2326 gonorrhoisch infiziert wared. Mithin 2 pCt. neu in Behandlung tretende Geschlechtskranke. Diese Angaben erreichen die Wirklichkeit nicht, da die von Kur-pfuschern aller Art behandelten Kranken ausfallen, auch nicht alle Mediziner gemeldet haben. Ausschliessiich auf die dort ansässigen Geschlechtskranken entfielen 71 pCt. männliche gegen 29 pCt. weibliche Patienten. Noch mehr zu Ungunsten der Manner verschiebt sich das Verhältnis, wenn auch die Militär-, Marine- und Schiñ $1 / 8$ werft-Bevölkerung der Hafenstadt in Betracht gezogen wird. Dazu kommt, dass mindestens 2,2 pCt. aller Geburten mit angeborener Syphilis behaftet waren. Die Zahl der extragenitalen Infektionen scheint in den letzten Jahren zurückgegangen zu sein, ebenso die gonor-rhoischen Infektionen des Auges. Auch war es unter den einigermassen übersichtlichen 
Ortsverhältnissen möglich, einen sanitären Vergleich zwischen geheimer und kontrollierter Prostitution anzustellen. Dieser Vergleich ist naeh den gemachten Erhebungen ganz wesentlich zugunsten einer humanen gesetzlichen Regelung der Prostitution ausgefallen. 